\title{
AVALIAÇÃO DA UTILIZAÇÃO DE COMPOSTO ORGÂNICO PRODUZIDO A PARTIR DOS RESÍDUOS DE FÁBRICA DE CELULOSE QUANTO AOS NÍVEIS DE ORGANOCLORADOS E METAIS ${ }^{1}$
}

Marcos Antônio de Souza Lima Guerra², Claudio Mudado Silva³, Ana Augusta Passos Rezende ${ }^{4}$, Hygor Aristides Rossoni ${ }^{5}$, Sebastião Tomas Carvalho ${ }^{6}$ e Ismarley Horta Lage Morais ${ }^{7}$

\begin{abstract}
RESUMO - Lodos gerados em Estações de Tratamento de Efluentes de fábricas de celulose kraft branqueada podem conter metais pesados e compostos organoclorados mesmo para fábricas que possuam sequências de branqueamento ECF (livres de cloro elementar), devido à presença de dióxido de cloro. Neste trabalho, buscou-se avaliar o potencial de absorção de metais pesados e compostos organoclorados (PCDD e PCDF) por Brassica juncea, utilizando como substrato o composto orgânico constituído por lodo biológico de uma Estação de Tratamento de Efluentes por lodos ativados misturado com cascas de eucalipto de uma fábrica de celulose brasileira. As plantas de Brassica juncea foram cultivadas em vasos de 0,63 $\mathrm{dm}^{3} \mathrm{em}$ casa de vegetação, durante 73 dias. Os seguintes parâmetros foram monitorados no substrato e nas plantas: $\mathrm{Fe}, \mathrm{Mn}, \mathrm{Pb}, \mathrm{Ni}, \mathrm{Cu}, \mathrm{Zn}, \mathrm{Cr}$, $\mathrm{Cl}^{-}$, carbono orgânico total e AOX (compostos organo-halogenados adsorvíveis). Os níveis de metais pesados presentes no solo após a aplicação do composto ficaram dentro de faixas permissíveis da legislação brasileira para aplicação de composto orgânico para uso agrícola. Traços de PCDD e PCDF foram encontrados no substrato em concentrações menores do que $0,5 \mathrm{ng} \mathrm{I-TEQ/kg} \mathrm{(ppt).} \mathrm{Tais} \mathrm{valores} \mathrm{ficaram} \mathrm{abaixo} \mathrm{de} 17 \mathrm{ng}$ I-TEQ/kg, considerado o limite mais exigente de qualidade de compostos adotados na Alemanha e no Canadá.
\end{abstract}

Palavras-chave: Composto, Celulose Kraft, Metais e Organoclorados.

\section{EVALUATION OF THE USE OF ORGANIC COMPOST PRODUCED FROM PULP MILL RESIDUES IN REGARDS TO CHLORINATED ORGANIC COMPOUNDS AND METALS}

\begin{abstract}
Bleached kraft pulp mill sludge may present traces of heavy metals and organochorine compounds even when ECF (elemental chorine free) bleaching sequences are used due to the presence of chlorine dioxide. The objective of this work was to evaluate the absorption potential of heavy metals and organochlorine compounds (PCDD and PCDF) by Brassica juncea when bio-sludge of an activated sludge plant and organic compost prepared from bio-sludge mixed with Eucalyptus bark of a bleached kraft pulp mill. The plants were cultivated in $0.63 \mathrm{dm}^{3}$ pots in greenhouses during 73 days. The following parameters were monitored in the substrate and in the plants: $\mathrm{Fe}, \mathrm{Mn}, \mathrm{Pb}, \mathrm{Ni}, \mathrm{Cu}, \mathrm{Zn}, \mathrm{Cr}, \mathrm{Cl}^{-}$, total organic carbon and $\mathrm{AOX}$ (adsorbable organic halogen compounds). Content of heavy metals in the soil after compost application was within the Brazilian legal standards for compost application. Traces of PCDD e PCDF were found in the substrate at concentrations lower than $0.5 \mathrm{ng} I-T E Q / \mathrm{kg}$ (ppt). These values were lower than the highest quality criteria of $17 \mathrm{ng} I-T E Q / \mathrm{kg}$ from Canada and Germany.
\end{abstract}

Keywords: Compost, kraft pulp and Solid waste.

\footnotetext{
${ }^{1}$ Recebido em 11.05.2010 e aceito para publicação em 29.03.2012.

${ }^{2}$ Mestre em Ciência Florestal pela Universidade Federal de Viçosa, UFV, Brasil.

${ }^{3}$ Departamento de Engenharia Florestal, Universidade Federal de Viçosa, UFV, Brasil. E-mail: <mudado@ufv.br>.

${ }^{4}$ Departamento de Engenharia Civil, Universidade Federal de Viçosa, UFV, Brasil. E-mail: <ana.augusta@ufv.br>.

${ }^{5}$ Instituto de Ciências Exatas e Tecnológicas, Universidade Fedral de Viçosa, UFV, Brasil. E-mail: $<$ rossoni@ufv.br>.

${ }^{6}$ Celulose Nipo Brasileira, CENIBRA, Brasil. E-mail: <sebastiao.carvalho@cenibra.com.br>.

${ }^{7}$ Mestre em Ciència Florestal pela Universidade Federal de Viçosa, UFV, Brasil. E-mail: <ismarleylage@gmail.com>.
} 


\section{INTRODUÇÃO}

Nos últimos anos, a produção de celulose kraft branqueada vem crescendo significativamente no Brasil, e com isso aumenta-se a preocupação com os aspectos ambientais relativos a essa tipologia industrial. $\mathrm{O}$ processo industrial gera elevada quantidade de resíduos sólidos que deve ser adequadamente gerenciada. A utilização de resíduos aplicados diretamente no solo (CUNHA, A. M. et al., 2006) ou para outros fins (MACHADO, C. C. et al., 2007) tem sido extensivamente estudada.

O processo de compostagem vem sendo largamente utilizado por empresas brasileiras como forma de tratamento dos resíduos sólidos orgânicos, transformando-os em húmus, que podem ser utilizados diretamente nos plantios florestais como condicionadores de solo. No entanto, devido ao histórico de formação de compostos organoclorados no branqueamento das indústrias de celulose, há dúvidas quanto à segurança ambiental dessa prática. Embora se saiba que a formação desses organoclorados está associada à utilização do cloro elementar no branqueamento da celulose. Atualmente, a maioria das indústrias brasileiras substituiu completamente o cloro elementar $\left(\mathrm{Cl}_{2}\right)$ pelo dióxido de cloro $\left(\mathrm{ClO}_{2}\right)$ em sequências denominadas ECF (Elemental Chlorine Free), favorecendo a diminuição da formação desses compostos organoclorados.

O objetivo deste trabalho foi verificar a qualidade do composto orgânico gerado a partir de cascas de eucalipto e lodo biológico de uma fábrica de celulose kraft branqueada, quanto aos níveis de organoclorados (AOX, dioxinas e furanos) e metais pesados, determinando-se a capacidade de absorção desses elementos pela planta Brassica juncea (mostarda lisa ou mostarda de folhas). Essa planta foi escolhida pelo seu alto potencial de acumulação de metais, sendo bastante estudada para fins de biorremediação (CLEMENTE et al., 2005; REISINGER et al., 2008).

\section{MATERIAL E MÉTODOS}

O experimento foi conduzido em casa de vegetação durante 73 dias, compreendendo 39 parcelas experimentais, constituídas de vasos contendo amostras de material de 13 diferentes misturas de substratos (solo, lodo biológico e composto orgânico) (Tabela 1). Como cultivar, utilizaram-se as plantas Brassica juncea, montadas no delineamento inteiramente casualizado com três repetições. Os dados foram interpretados por meio de análise de variância e as médias dos tratamentos (misturas dos substratos), comparadas pelo teste de Tukey a 5\% de probabilidade.

O solo utilizado no experimento foi classificado como Latossolo Vermelho-Amarelo. Os substratos, o composto orgânico e o lodo biológico foram fornecidos pela empresa Celulose Nipo-Brasileira-CENIBRA. O primeiro foi produzido por um processo de compostagem em leiras estáticas a partir dos resíduos sólidos industriais (casca de eucalipto triturada e lodo biológico) e o segundo, retirado da linha de recirculação de lodo biológico do sistema de tratamento secundário de efluentes da empresa, por meio do processo de lodos ativados. Com os objetivos de aumentar a consistência a 15\% de sólidos e, consequentemente, reduzir a umidade, o lodo biológico foi submetido ao processo de filtração em laboratório.

Os transplantes das mudas de Brassica juncea foram feitos após 22 dias de semeadura e a colheita após 51 dias do transplante, cortadas rentes à superfície dos substratos. O conteúdo de matéria seca (parte aérea) foi determinado após a secagem em estufa de ventilação forçada de ar, na temperatura de $60^{\circ} \mathrm{C}$, até atingir peso constante. As amostras foram moídas e mineralizadas para análise química. A extração química das amostras de plantas foi feita via úmida em $0,5 \mathrm{~g}$ de material seco com ácido nítrico p.a. (65\%) e ácido perclórico p.a. (70\%), em que foram determinados $\mathrm{Fe}, \mathrm{Mn}, \mathrm{Pb}, \mathrm{Ni}, \mathrm{Cu}, \mathrm{Zn}$ e $\mathrm{Cr}$ por espectrofotometria de plasma-ICP(EMBRAPA, 1999).

Tabela 1 - Misturas dos substratos usados para cada tratamento (\% peso/peso).

Table 1 - Substrate mixture used in each treatment.

\begin{tabular}{cccccccccccccc}
\hline Tratamentos & 1 & 2 & 3 & 4 & 5 & 6 & 7 & 8 & 9 & 10 & 11 & 12 & 13 \\
\hline Solo (\%) & 100 & 99 & 90 & 75 & 50 & 25 & 0 & 99 & 90 & 75 & 50 & 25 & 0 \\
L. biológico (\%) & 0 & 1 & 10 & 25 & 50 & 75 & 100 & 0 & 0 & 0 & 0 & 0 & 0 \\
C. orgânico (\%) & 0 & 0 & 0 & 0 & 0 & 0 & 0 & 1 & 10 & 25 & 50 & 75 & 100 \\
\hline
\end{tabular}

Revista Árvore, Viçosa-MG, v.36, n.2, p.381-387, 2012 
Para a extração química dos substratos, utilizaram-se as metodologias descritas em Embrapa (1997) para os parâmetros mencionados. As concentrações de AOX foram determinadas segundo a Norma SCAN - W 9:89 (SCAN, 1989). Para as análises de dioxinas e furanos, as amostras foram enviadas para um laboratório especializado (Analytical Solutions), sendo analisado e quantificado por cromatografia gasosa acoplada à espectrometria de massas de alta resolução de diluições isotrópicas, utilizando-se as metodologias USEPA 1613 (USEPA, 1994).

\section{RESULTADOS}

Os resultados médios de três repetições das concentrações de metais em cada tratamento com lodo biológico e composto orgânico estão apresentados na Tabela 2.

A Tabela 3 apresenta os valores de concentração de metais pesados nas plantas.

Na Tabela 4 são mostrados os valores observados de carbono orgânico, $\mathrm{Cl}^{-}$e AOX no composto orgânico e no lodo biológico em cada tratamento.
Os valores dos compostos dibenzodioxinas policloradas (PCDDs) e dibenzofuranos policlorados (PCDFs), expressos como Equivalente Tóxico (EqT), nas amostras dos substratos, são reportados na Tabela 5.

\section{DISCUSSÃO}

De acordo com a Tabela 2, observa-se que os valores se encontram inferiores aos máximos permissíveis para aplicação na agricultura (500 mg Pb.kg ${ }^{-1}, 500$ mg Cu.kg-1, 1.500 mg Zn.kg ${ }^{-1}, 300$ mg Cr.kg ${ }^{-1}$ e 100 mg Ni.kg-1), propostos pelo Ministério da Agricultura, Pecuária e Abastecimento (BRASIL, 2002).

Deve-se, entretanto, considerar que a mobilidade desses metais no solo dependerá da forma química sob a qual o metal se apresenta e das características do solo como pH, teor de matéria orgânica, CTC e porcentagem de argila, que têm importância fundamental na mobilidade de cátions metálicos (LOPES et al., 2005).

Os metais Pb e Ni não foram possíveis de serem quantificados nos extratos obtidos. Isso pode significar que o extrator utilizado, Mehlich modificado (HCL 0,05 mol.L e $^{-1} \mathrm{H}_{2} \mathrm{SO}_{4}$ 0,025 mol.L ${ }^{-1}$ ), não removeu esses

Tabela 2 - Teores de metais observados em cada tratamento dos substratos.

Table 2 - Contents of the metals concentration in substrates.

\begin{tabular}{|c|c|c|c|c|c|c|c|}
\hline Dosagem & $\mathrm{Fe}$ & $\mathrm{Mn}$ & $\mathrm{Pb}$ & $\mathrm{Ni}$ & $\mathrm{Cu}$ & $\mathrm{Zn}$ & $\mathrm{Cr}$ \\
\hline \multicolumn{8}{|c|}{ Composto orgânico mg.kg ${ }^{-1}$} \\
\hline $100 \%$ solo & $22,13 \mathrm{c}$ & $2,90 \mathrm{f}$ & nd & nd & $0,37 \mathrm{a}$ & $0,95 \mathrm{~d}$ & $0,12 \mathrm{~b}$ \\
\hline 100\% Comp. & $119,92 \mathrm{a}$ & $149,62 \mathrm{a}$ & nd & nd & $0,37 \mathrm{a}$ & $4,83 \mathrm{a}$ & $0,56 \mathrm{a}$ \\
\hline $75 \%$ Comp. $+25 \%$ solo & $116,41 \mathrm{a}$ & $135,24 \mathrm{~b}$ & nd & nd & $0,33 \mathrm{ab}$ & $3,54 \mathrm{~b}$ & $0,48 \mathrm{ab}$ \\
\hline $50 \%$ Comp. $+50 \%$ solo & $99,58 \mathrm{ab}$ & $105,75 \mathrm{c}$ & nd & nd & $0,25 \mathrm{ab}$ & $2,45 \mathrm{c}$ & $0,22 \mathrm{ab}$ \\
\hline $25 \%$ Comp. $+75 \%$ solo & $96,81 \mathrm{ab}$ & $67,22 \mathrm{~d}$ & nd & nd & $0,24 \mathrm{ab}$ & $1,49 \mathrm{~cd}$ & $0,14 \mathrm{~b}$ \\
\hline $10 \%$ Comp. $+90 \%$ solo & $68,42 \mathrm{~b}$ & $26,49 \mathrm{e}$ & nd & nd & $0,16 \mathrm{~b}$ & $1,27 \mathrm{~d}$ & $0,21 \mathrm{ab}$ \\
\hline $1 \%$ Comp. $+99 \%$ solo & $32,85 \mathrm{c}$ & $1,90 \mathrm{f}$ & nd & nd & $0,17 \mathrm{~b}$ & $1,04 \mathrm{~d}$ & $0,13 \mathrm{~b}$ \\
\hline \multicolumn{8}{|c|}{ Lodo biológico mg.kg ${ }^{-1}$} \\
\hline $100 \%$ solo & $22,13 \mathrm{c}$ & $2,90 \mathrm{~d}$ & nd & nd & $0,37 \mathrm{~b}$ & $0,95 \mathrm{~b}$ & 0,12 \\
\hline 100\% Lodo Biológico & $76,53 \mathrm{~b}$ & $124,73 \mathrm{a}$ & nd & nd & $0,25 \mathrm{~b}$ & $7,59 \mathrm{a}$ & nd \\
\hline $75 \%$ Lodo $+25 \%$ solo & $167,97 \mathrm{a}$ & $34,72 \mathrm{~b}$ & nd & nd & $0,87 \mathrm{a}$ & $4,81 \mathrm{ab}$ & nd \\
\hline $50 \%$ Lodo $+50 \%$ solo & $79,89 \mathrm{~b}$ & $24,34 \mathrm{c}$ & nd & nd & $0,41 \mathrm{~b}$ & $2,07 \mathrm{~b}$ & nd \\
\hline $25 \%$ Lodo $+75 \%$ solo & $25,10 \mathrm{c}$ & $5,95 \mathrm{~d}$ & nd & nd & $0,07 \mathrm{c}$ & $0,68 \mathrm{~b}$ & nd \\
\hline $10 \%$ Lodo $+90 \%$ solo & $14,56 \mathrm{c}$ & $3,31 \mathrm{~d}$ & nd & nd & nd & $0,52 \mathrm{~b}$ & nd \\
\hline $1 \%$ Lodo $+99 \%$ solo & $15,31 \mathrm{c}$ & $1,19 \mathrm{~d}$ & nd & nd & nd & $0,35 \mathrm{~b}$ & nd \\
\hline
\end{tabular}

As médias seguidas de pelo menos uma mesma letra na vertical, dentro de cada grupo de amostra, não diferem entre si, estatisticamente em nível de 5\% de probabilidade, pelo teste de Tukey. nd = não detectados teores abaixo do limite de detecção pelo método analítico empregado (0,001 mg. $\left.\mathrm{kg}^{-1}\right)$; e ns = efeito não significativo, pelo teste $\mathrm{F}$ a $5 \%$ de probabilidade. 
Tabela 3 - Teores de metais observados nas plantas dentro de cada tratamento. Table 3 - Concentration of metals in metals concentration in the plants.

\begin{tabular}{|c|c|c|c|c|c|c|c|}
\hline Dosagem & $\mathrm{Fe}$ & $\mathrm{Mn}$ & $\mathrm{Pb}$ & $\mathrm{Ni}$ & $\mathrm{Cu}$ & $\mathrm{Zn}$ & $\mathrm{Cr}$ \\
\hline \multicolumn{8}{|c|}{ Composto orgânico $\mu \mathrm{g} \cdot \mathrm{kg}^{-1}$} \\
\hline $100 \%$ solo & $\mathrm{sm}$ & sm & sm & $\mathrm{sm}$ & $\mathrm{sm}$ & sm & sm \\
\hline $100 \%$ Comp. Org. & $5,581 \mathrm{a}$ & $0,101 \mathrm{a}$ & $0,019 \mathrm{a}$ & $0,104 \mathrm{a}$ & $0,006 \mathrm{a}$ & $0,207 \mathrm{a}$ & $0,186 \mathrm{a}$ \\
\hline $75 \%$ Comp. $+25 \%$ solo & $0,536 \mathrm{~b}$ & $0,019 \mathrm{c}$ & $0,004 \mathrm{~b}$ & $0,005 \mathrm{~b}$ & $0,003 \mathrm{bc}$ & $0,025 \mathrm{~b}$ & $0,004 \mathrm{~b}$ \\
\hline $50 \%$ Comp. $+50 \%$ solo & $0,432 \mathrm{~b}$ & $0,019 \mathrm{c}$ & $0,004 \mathrm{~b}$ & $0,001 \mathrm{~b}$ & $0,002 \mathrm{bc}$ & $0,027 \mathrm{~b}$ & $0,002 \mathrm{~b}$ \\
\hline $25 \%$ Comp. $+75 \%$ solo & $0,481 \mathrm{~b}$ & $0,026 \mathrm{bc}$ & $0,008 \mathrm{~b}$ & $0,001 \mathrm{~b}$ & $0,002 \mathrm{c}$ & $0,027 \mathrm{~b}$ & $0,002 \mathrm{~b}$ \\
\hline $10 \%$ Comp. $+90 \%$ solo & $1,470 \mathrm{~b}$ & $0,064 \mathrm{ab}$ & $0,006 \mathrm{~b}$ & $0,003 \mathrm{~b}$ & $0,004 \mathrm{~b}$ & $0,026 \mathrm{~b}$ & $0,005 \mathrm{~b}$ \\
\hline $1 \%$ Comp. $+99 \%$ solo & $\mathrm{sm}$ & $\mathrm{sm}$ & sm & $\mathrm{sm}$ & $\mathrm{sm}$ & sm & sm \\
\hline \multicolumn{8}{|c|}{ Lodo biológico $\mu \mathrm{g} \cdot \mathrm{kg}^{-1}$} \\
\hline $100 \%$ solo & $\mathrm{sm}$ & $\mathrm{sm}$ & $\mathrm{sm}$ & $\mathrm{sm}$ & $\mathrm{sm}$ & $\mathrm{sm}$ & $\mathrm{sm}$ \\
\hline $100 \%$ Lodo & $\mathrm{sm}$ & $\mathrm{sm}$ & $\mathrm{sm}$ & $\mathrm{sm}$ & $\mathrm{sm}$ & $\mathrm{sm}$ & sm \\
\hline $75 \%$ Lodo $+25 \%$ solo & $\mathrm{sm}$ & $\mathrm{sm}$ & $\mathrm{sm}$ & $\mathrm{sm}$ & $\mathrm{sm}$ & $\mathrm{sm}$ & $\mathrm{sm}$ \\
\hline $50 \%$ Lodo $+50 \%$ solo & $0,354 \mathrm{c}$ & $0,129 \mathrm{~ns}$ & $0,015 \mathrm{~ns}$ & $0,003 \mathrm{~b}$ & $0,003 \mathrm{~ns}$ & $0,033 \mathrm{~ns}$ & $0,004 \mathrm{~b}$ \\
\hline $25 \%$ Lodo $+75 \%$ solo & $1,898 \mathrm{~b}$ & $0,156 \mathrm{~ns}$ & $0,023 \mathrm{~ns}$ & $0,007 \mathrm{~b}$ & $0,005 \mathrm{~ns}$ & $0,030 \mathrm{~ns}$ & $0,010 \mathrm{~b}$ \\
\hline $10 \%$ Lodo $+90 \%$ solo & $3,002 \mathrm{a}$ & $0,123 \mathrm{~ns}$ & $0,015 \mathrm{~ns}$ & $0,013 \mathrm{a}$ & $0,005 \mathrm{~ns}$ & $0,048 \mathrm{~ns}$ & $0,026 \mathrm{a}$ \\
\hline $1 \%$ Lodo $+99 \%$ solo & $\mathrm{sm}$ & sm & $\mathrm{sm}$ & $\mathrm{sm}$ & $\mathrm{sm}$ & $\mathrm{sm}$ & sm \\
\hline
\end{tabular}

Médias seguidas pelas mesmas letras na vertical, dentro de cada grupo de amostra, não diferem estatisticamente a 5\%, pelo teste de Tukey. nd = não detectados teores abaixo do limite de detecção pelo método analítico empregado. $\mathrm{sm}=$ massa seca foliar insuficiente, abaixo de $0,1 \mathrm{~g}$.

ns = efeito não significativo, pelo teste $\mathrm{F}$ a 5\% de probabilidade.

Tabela 4 - Concentrações de carbono orgânico, $\mathrm{Cl}^{-}$e AOX observados em cada tratamento dos substratos.

Table 4 - Organic carbon, $\mathrm{Cl}^{-}$and $\mathrm{AOX}$ in each substrate treatment.

\begin{tabular}{|c|c|c|c|}
\hline Dosagem & Carbono orgânico & $\mathrm{Cl}^{-}$ & AOX \\
\hline \multicolumn{4}{|c|}{ Composto orgânico } \\
\hline & g. $\mathrm{kg}^{-1}$ & g. $\mathrm{kg}^{-1}$ & mg.kg ${ }^{-1}$ \\
\hline $100 \%$ solo & $81,32 \mathrm{c}$ & $2,464 \mathrm{~ns}$ & $25,23 \mathrm{~cd}$ \\
\hline $100 \%$ Comp. Org. & $255,89 \mathrm{a}$ & $1,933 \mathrm{~ns}$ & $68,54 \mathrm{a}$ \\
\hline $75 \%$ Comp. $+25 \%$ solo & $210,12 \mathrm{ab}$ & $2,197 \mathrm{~ns}$ & $46,47 \mathrm{~b}$ \\
\hline $50 \%$ Comp. $+50 \%$ solo & $177,48 \mathrm{~b}$ & $2,233 \mathrm{~ns}$ & $32,29 \mathrm{c}$ \\
\hline $25 \%$ Comp. $+75 \%$ solo & $110,05 \mathrm{c}$ & $2,389 \mathrm{~ns}$ & $23,70 \mathrm{c}$ \\
\hline $10 \%$ Comp. $+90 \%$ solo & $91,35 \mathrm{c}$ & $2,481 \mathrm{~ns}$ & $18,71 \mathrm{de}$ \\
\hline $1 \%$ Comp. $+99 \%$ solo & $75,37 \mathrm{c}$ & $2,064 \mathrm{~ns}$ & $14,26 \mathrm{e}$ \\
\hline \multicolumn{4}{|c|}{ Lodo biológico } \\
\hline & g.kg ${ }^{-1}$ & g. $\mathrm{kg}^{-1}$ & $\mathrm{mg} \cdot \mathrm{kg}^{-1}$ \\
\hline $100 \%$ solo & $81,32 \mathrm{c}$ & $2,464 \mathrm{~ns}$ & $25,23 \mathrm{~cd}$ \\
\hline $100 \%$ Lodo Biológico & $759,20 \mathrm{a}$ & $2,208 \mathrm{~ns}$ & $1214,63 \mathrm{a}$ \\
\hline $75 \%$ Lodo $+25 \%$ solo & $205,35 \mathrm{~b}$ & $2,465 \mathrm{~ns}$ & $138,86 \mathrm{~b}$ \\
\hline $50 \%$ Lodo $+50 \%$ solo & $85,01 \mathrm{c}$ & $2,285 \mathrm{~ns}$ & $64,50 \mathrm{c}$ \\
\hline $25 \%$ Lodo $+75 \%$ solo & $81,24 \mathrm{c}$ & $1,897 \mathrm{~ns}$ & $23,00 \mathrm{~cd}$ \\
\hline $10 \%$ Lodo $+90 \%$ solo & $64,08 \mathrm{c}$ & $2,200 \mathrm{~ns}$ & $25,96 \mathrm{~cd}$ \\
\hline $1 \%$ Lodo $+99 \%$ solo & $57,67 \mathrm{c}$ & $2,054 \mathrm{~ns}$ & $8,06 \mathrm{~d}$ \\
\hline
\end{tabular}

Médias seguidas pelas mesmas letras na vertical, dentro de cada grupo de amostra, não diferem estatisticamente a 5\%, pelo teste de Tukey. $\mathrm{ns}=$ efeito não significativo, pelo teste $\mathrm{F}$ a $5 \%$ de probabilidade.

Revista Árvore, Viçosa-MG, v.36, n.2, p.381-387, 2012 
Tabela 5 - Valores de Equivalente Tóxicos (pg EqT/kg). Table 5 - Toxic Equivalent (pg TEQ/kg).

\begin{tabular}{lc}
\hline Amostras & Valores \\
\hline Solo 100\% (substrato) & 1,6 \\
Composto orgânico 100\% (substrato) & 400 \\
Lodo biológico 100\% (substrato) & 130 \\
Lodo biológico 25\% (substrato) & 2,4 \\
Lodo biológico 25\% (foliar) & nd \\
Composto orgânico 100\% (foliar) & nd \\
Comercializada em Viçosa (foliar - testemunha) & nd \\
\hline
\end{tabular}

nd Não detectado.

metais em quantidades que pudessem ser medidas pelo equipamento de absorção atômica. O Cr não quantificado nos tratamentos com adição de lodo biológico apresentou o maior valor ( $\left.0,56 \mathrm{mg} . \mathrm{kg}^{-1}\right)$ para o tratamento com $100 \%$ de composto. Baixos teores de $\mathrm{Cu}$ (abaixo de 0,87 mg.kg-1) foram encontrados nos tratamentos utilizando composto orgânico e lodo biológico como substrato, não sendo detectados nos tratamentos com 1 e $10 \%$ de lodo biológico (Tabela 2).

Os maiores valores encontrados de Fe nos substratos foram de 167,97 mg.kg ${ }^{-1}$ e 119,92 mg.kg-1, no tratamento com $75 \%$ de lodo biológico e no tratamento com $100 \%$ de composto orgânico, respectivamente. Os maiores valores de Mn disponíveis no substrato foram de 149,62 mg. $\mathrm{kg}^{-1}$ no tratamento com $100 \%$ de composto orgânico e 124,73 mg. kg ${ }^{-1}$ no tratamento com 100\% de lodo biológico. O maior valor de $\mathrm{Zn}$ encontrado nos tratamentos foi de 7,59 mg. $\mathrm{kg}^{-1}$.

Os valores de metais disponíveis encontravam-se muito abaixo das concentrações normais de metais nos solo, informados por Santos (2005). De acordo com esse autor, para as concentrações críticas no solo atingirem níveis de toxicidade, os valores encontrados teriam que ser superiores aos seguintes: 70-400 mg Zn.kg-1, 60-125 mg Cu.kg-1, 3-8 mg Cd.kg-1, 100 mg Ni.kg ${ }^{-1}$, 100-400 mg Pb.kg-1.

A baixa concentração de metais pesados apresentados no composto orgânico e no lodo biológico foi devida às características da indústria de celulose e papel, onde esses elementos se encontravam presentes na anatomia da madeira e, em alguns casos restritos, podem ser gerados por meio de corrosões localizadas na indústria, sendo carreados para a estação de tratamento de efluentes e retirados junto com o lodo biológico. Posteriormente, esse lodo seria utilizado para a produção do composto orgânico junto com as cascas de eucalipto.

A capacidade das plantas em acumular elevados níveis de metais pesados (hiperacumulação) foi observada em várias espécies. Essa hiperacumulação depende de vários fatores, como: da capacidade genética e da bioviabilidade do metal pela planta (BLAYLOCK et al., 1997).

Segundo Santos (2005), a Brassica juncea é uma das espécies herbáceas de plantas mais tolerantes ao excesso de metais no solo, podendo acumular e translocar concentrações acima de $10.000 \mathrm{mgkg}^{-1} \mathrm{em}$ reduzidas quantidades de solo (EPSTEIN et al.,1999; CLEMENTE et al., 2005; REISINGER et al.,2008).

Os resultados médios de três repetições para concentrações de metais nas amostras foliares indicaram que as plantas do tratamento com $100 \%$ de composto orgânico apresentaram os maiores valores entre todos os tratamentos propostos, como 5,581 $\mu \mathrm{g} \mathrm{Fe.} \mathrm{kg}^{-1}, 0,019$ $\mu \mathrm{g}$ Pb.kg ${ }^{-1}, 0,104 \mu \mathrm{g} \mathrm{Ni.kg}{ }^{-1}, 0,006 \mu \mathrm{g}$ Cu. $\mathrm{kg}^{-1}, 0,207 \mu \mathrm{g}$ Zn.kg-1 e 0,186 $\mu$ g Cr.kg ${ }^{-1}$ (Tabela 3). Alguns tratamentos, como 1\% de composto orgânico, 100\% de solo e 1\% de lodo biológico, não tiveram nutrientes necessários para o desenvolvimento das plantas, não obtendo massa seca foliar necessária para amostragem (abaixo de 0,1 g). Os tratamentos com 75\% e 100\% de lodo biológico, por efeito de toxicidade, não apresentaram desenvolvimento das plantas. As concentrações de metais encontradas nas amostras foliares foram muito inferiores aos valores típicos de serem encontrados em plantas, reportados por Santos (2005), que descreveu os valores normais de serem encontrados em plantas, como: 1 - 400 mg Zn.kg-1, 5 - 20 mg Cu. kg-1, 20 - 300 mg Mn.kg-1 , 0,1 - 2,4 mg Cd.kg-1 , 0,02 - 5 mg Ni.kg ${ }^{-1}$

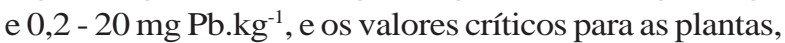
como: 100 - 400 mg Zn.kg ${ }^{-1}, 20$ - 100 mg Cu.kg ${ }^{-1}, 300$ - 500 mg Mn.kg-1 , 5 - 30 mg Cd.kg ${ }^{-1}, 10$ - 100 mg Ni.kg-1 e 30 - 300 mg Pb. kg-1.

É importante ressaltar que o equipamento para a determinação de metais, utilizado nas amostras foliares, foi a espectrofotometria de plasma, o qual possui nível de detecção melhor do que a espectrofotometria de absorção atômica, que foi empregada nas determinações de metais nas amostras dos substratos.

Devido ao baixo nível de metais encontrados nos substratos e nas plantas, a correlação para se avaliar a absorção desses elementos pelas plantas foi muito

Revista Árvore, Viçosa-MG, v.36, n.2, p.381-387, 2012 
baixa. Isso significa que os teores encontrados na análise foliar não representam fator restritivo ao consumo humano.

O parâmetro global que mede o teor total de material organoclorado em efluentes líquidos é o Halógenos Orgânicos Adsorvíveis (AOX). Este engloba os mais de 300 compostos clorados já identificados em efluentes de branqueamento (MCKAGUE et al., 1998), além do material clorado polimérico.

As dibenzodioxinas policloradas (PCDD), os dibenzofuranos policlorados (PCDF) e as bifenilas policloradas (PCB) estão entre os compostos organoclorados mais tóxicos e persistentes já conhecidos. O mais estudado e mais tóxico nesse grupo de compostos é o 2,3,7,8-tetraclorodibenzo-p-dioxina (TCDD), muitas vezes chamado simplesmente de dioxina, que representa a referência para o grupo. Esses compostos são hidrofóbicos, resistem ao metabolismo, persistem no meio ambiente e são propensos a bioacumular em tecidos adiposos de animais (USEPA, 2000). A formação de dioxinas e furanos requer, necessariamente, uma fonte de cloro, uma fonte de matéria orgânica e um ambiente térmico ou quimicamente reativo (CABRITA, 2003).

A Tabela 4 apresenta os valores observados de carbono orgânico, $\mathrm{Cl}^{-}$e AOX em cada tratamento. Os valores de Halógenos Orgânicos Adsorvíveis (AOX), observados nos substratos de composto orgânico, estão abaixo do limite estabelecido pela Comunidade Europeia para aplicação do composto no solo (SEPA, 1997), de 500 mg. $\mathrm{kg}^{-1}$. O tratamento com $100 \%$ de lodo biológico, o valor de AOX encontrado (1.214 mg. $\mathrm{kg}^{-1}$ ) superou esse limite. Em relação aos valores de $\mathrm{Cl}^{-}$, estes não apresentaram variação significativa na análise de variância aplicada pelo teste F a 5\% de probabilidade. Assim, o menor valor encontrado foi $1,9 \mathrm{~g} \cdot \mathrm{kg}^{-1}$ no tratamento com $25 \%$ de lodo biológico e o maior, 2,5 g. $\mathrm{kg}^{-1}$ no tratamento com $10 \%$ de composto orgânico.

Em relação aos valores de $\mathrm{Cl}^{-}$, estes não apresentaram efeitos significativos na análise de variância aplicada pelo teste $\mathrm{F}$ a 5\% de probabilidade.

O padrão mais restrito reportado em EqT de PCDD e PCDF, 17 ng/kg, em composto orgânico para uso agrícola é o estabelecido na Província de Quebec, no Canadá, a partir do padrão alemão (GROENEVELD; HEBERT, 2003). Dessa forma, constatou-se que o maior valor de EqT encontrado (0,4 ng EqT/kg) no composto orgânico foi menor do que o mais restritivo padrão canadense e alemão. A empresa Celulose Nipo-Brasileira-CENIBRA utiliza em seu processo de branqueamento da pasta celulósica apenas dióxido de cloro em sequências denominadas ECF, portanto não se espera a geração de dioxinas e furanos.

Conforme valores reportados na Tabela 5, nas amostras foliares não foram encontrados valores de EqT para dioxinas e furanos. Estudos da fisiologia baseados em nutrição de plantas não confirmaram o transporte de compostos com estruturas complexas como a dioxina e furanos, através das raízes até as folhas, ou seja, somente haverá contaminação das folhas através de contato direto (RIZZARDI et al., 2003).

\section{CONCLUSÕES}

As concentrações de metais e os valores de AOX e EqT encontrados nas amostras dos substratos compostos por diferentes proporções de solo, composto orgânico e lodo biológico foram inferiores aos limites máximos para uso agrícola propostos pelo Ministério da Agricultura, Pecuária e Abastecimento e adotados no Canadá e na Alemanha, respectivamente. As concentrações de metais encontrados no material foliar apresentaram valores inferiores aos limites críticos, não sendo constatados valores de dioxinas e furanos no material foliar. Segundo os resultados, não haveria nenhuma restrição, quanto a esses parâmetros, para a utilização agrícola do composto orgânico produzido a partir da casca de eucalipto e lodo biológico.

\section{AGRADECIMENTOS}

À Empresa Celulose Nipo-Brasileira-CENIBRA; à FAPEMIG; e ao CNPq, pelo apoio na execução dos trabalhos.

\section{REFERÊNCIAS}

BLAYLOCK, M. J. et al. Enhanced accumulation of $\mathrm{Pb}$ in Indian mustard by soil-applied chelating agents.Environmental Science and Technology, v.31, p.860-865, 1997.

BRASIL. Ministério da Agricultura Pecuária e Abastecimento e Empresa Brasileira de Pesquisa Agropecuária Recomendações técnicas para o uso agrícola do composto de lixo urbano no estado de São Paulo. Campinas: 2002. (Embrapa, Circular Técnica, 3). 
CABRITA, I. et al. Formação e destruição de dioxinas em processos de combustão e cocombustão. In: ENCONTRO NACIONAL DE DIOXINAS E COMPOSTOS SIMILARES, 1., 2003, Portugal. Anais...Portugal: 2003.

CLEMENTE, R.; WALKER, D. J.; BERNAL, M. P. Uptake of heavy metals and As by Brassica juncea grown in a contaminated soil in Aznalcóllar (Spain): The effect of soil amendments. Environmental Pollution, v.138, n.1, p.46-58, 2005.

CUNHA, A. M. et al. Efeito de diferentes substratos sobre o desenvolvimento de mudas de Acácia sp. Revista Árvore, v.30, n.2, p.207-214, 2006.

EMPRESABRASILEIRADE PESQUISA AGROPECUÁRIA - EMBRAPA. Centro Nacional de Pesquisa de Solos. Manual de métodos de análises do solo. 2.ed. Rio de Janeiro: 1997. 247p.

EMPRESA BRASILEIRA DE PESQUISA

AGROPECUÁRIA - EMBRAPA. Embrapa Informática Agropecuária. Manual de análises químicas de solos, plantas e fertilizantes. Brasília: Embrapa - Comunicação para Transferência de Tecnologia, 1999. 370p.

EPSTEIN, A. L. et al. EDTA and Pb-EDTA accumulation in Brassica juncea grown in $\mathrm{Pb}-$ amended soil. Plant and Soil, v. 208, n.1, p. 87-94, 1999.

GROENEVELD, E.; HEBERT, M. Dioxins, furans, PCBs and PAHs in eastern Canada compost. Québec: Ministère de l'Environnement du Québec, Direction du Milieu Rural, Service de L'assainissement Agricole et des Activités de Compostage, 2003.

LOPES, J. C. et al. Produção de alface com doses de lodo de esgoto. Horticultura Brasileira, v.23, n.1, p. 143-147, 2005.

MACHADO, C. C. et al. Caracterização tecnológica de misturas solo-grits para pavimentos de estradas florestais: influência do tratamento térmico do grits na resistência mecânica das misturas. Revista Árvore, , v.31, n.3, p.487-494, 2007.
MCKAGUE, A. B.; CARLBERG, G. Effluent characteristics and composition. In: DENCE, C.; REEVE, D. W. (Eds). Pulp bleaching principles and practice. Atlanta: Tappi Press, 1998.

REISINGER S. et al. Heavy metal tolerance and accumulation in indian mustard (Brassica Juncea L.) expressing bacterial ã-glutamylcysteine synthetase or glutathione synthetase;

International Journal of Phytoremediation, v.10, n.5, p.440-454, 2008.

RIZZARDI, M. A. et al. Ação de herbicidas sobre mecanismos de defesa das plantas aos patógenos. Ciência Rural, v.33, n.5, p.957-965, 2003.

SANTOS, G. C. G. Comportamento de B, Zn, Cu, Mn e Pb em solo contaminado sob cultivo de plantas e adição de fontes de matéria orgânica como amenizantes do efeito tóxico. 2005. 98f. Tese (Doutorado em Solos e Nutrição de Plantas) Escola Superior de Agricultura Luiz de Queiroz, Piracicaba, 2005.

SCANDINAVIAN PULP, PAPER AND BOARD. Testing Committee. Stockholm: Norma SCAN W9:1989.

SWEDISH ENVIRONMENTAL PROTECTION AGENCY - SEPA. Compost quality and potential for use. AFR- 154, Stockholm: 1997.

UNITED STATES ENVIRONMENTAL PROTECTION AGENCY - USEPA. Method 1613.

Tetra- through octa-chlorinated dioxins and furans by isotope dilution HRGC/HRMS. Washington. D.C.: Office of Water Engineering and Analysis Division, 1994.

UNITED STATES ENVIRONMENTAL PROTECTION AGENCY - USEPA. Draft exposure and human health reassessment of $2,3,7,8$ tetrachlorodibenzo-p-dioxin (TCDD) and related compounds. EPA/600/P-00/001. Washington. D.C.: National Center for Environmental Assessment, September, 2000. 
